\title{
Design and Evaluation of Dual-Band Antennas Aimed for Contactless Health Monitoring Radar
}

\author{
Gokarna Pandey ${ }^{1}$, Ping Jack Soh ${ }^{1,2}$, Marco Mercuri ${ }^{1}$, Guy A. E. Vandenbosch ${ }^{1}$, Dominique \\ Schreurs ${ }^{1}$ \\ ${ }^{1}$ Div. ESAT-TELEMIC, Katholieke Universiteit Leuven, 3001 Leuven, Belgium \\ ${ }^{2}$ Sch. of Computer \& Communication Eng., Universiti Malaysia Perlis, 02000 Perlis, Malaysia \\ E-mail: gokarna063@gmail.com
}

\begin{abstract}
This paper presents the evaluation of two antenna designs aimed for a Step Frequency Continuous Wave (SFCW) radar capable of non-invasive fall detection in a pseudo-static radar configuration. The performance of a dual-band rectangular planar monopole is compared against a conductor-backed coplanar waveguide (CBCPW) fed bowtie slot antenna in free space. Two elements of the better performing CBCPW fed bow-tie antenna are then combined, separated by a metal plate, and evaluated on the level of SFCW radar operation. It is shown that the performance of the novel CBCPW fed bow-tie slot antenna is superior compared to the rectangular planar monopole antenna in terms of person scanning capability.
\end{abstract}

Index Terms - biomedical applications, bow-tie antenna, dual-band antenna, SFCW radar.

\section{INTRODUCTION}

Falls are the leading cause of injury for persons over the age of 65 years. Victims experiencing a home fall event and who remain undetected for more than one hour may suffer from many medical complications, with half of them dying within six months. In order to provide immediate assistance, it is crucial to detect falls as soon as they occur. A contactless health monitoring system using SFCW radar which detects the falls and alerts the caregiver for assistance is ideal for such purpose [1]. To effectively detect falls, multiple SFCW radar sensors can be integrated into a smart wireless sensor network (WSN). Zigbee is considered to be the best option for this WSN considering its energy-efficiency [2].

The SFCW radar transmits $N$ coherent $\mathrm{CW}$ pulses which frequencies are increased from pulse to pulse by a fixed increment $\Delta f$. The measured phase variations among transmitted and reflected $\mathrm{CW}$ pulses are used to determine the person's location and his/her speed [1]. The reflected information is also used to distinguish a fall event from normal movements. Depending on the absolute distance between the target and the antenna, the signal reflected from the human body is expected to be degraded by more than $30 \mathrm{~dB}$ relative to the transmitted signal. To detect the weaker reflected signals, efficient SFCW radar's front-end is necessary, facilitated by a careful design procedure. This front-end may be designed in two configurations: (1) using a two-antenna configuration - referred to as pseudo static configuration, or (2) using a single antenna with a circulator. In this work, the former setup is utilized to evaluate two antenna topologies: (1) a dual-band rectangular planar monopole (RPM), and (2) a dual-band bow-tie slot antennas (BSA), both radiating at WSN and radar operating frequencies. Due to the antennas' close proximity, this configuration is expected to mainly suffer from antenna mutual coupling, which must be kept under $-30 \mathrm{~dB}$ to ensure proper signal reception.

Wideband planar monopole antennas with different radiating patches have been recently reported [3-5]. Wide band CPW fed bow-tie slot antennas with inductively coupled slot [6] and narrow band antennas for RFID tag [7] have been also proposed. To our knowledge, the compact two-antenna configuration for simultaneous transmit and receive operation using a unidirectional dualband bowtie slot is yet to be investigated. In addition, the SFCW radar, which consists of the radar module, the Zigbee module, and a microcontroller [1], is evaluated using these two antenna topologies. In section II, the antenna designs and dimensions are presented. Their simulation and measurement results are explained in section III, prior to practical radar evaluations detailed in section IV.

\section{ANTENNA DESIGN}

The considered SFCW radar requires a bandwidth of at least $1 \mathrm{GHz}$. Initially, the RPM is designed for operation from 4-5 GHz, which was later changed to 6-7 GHz, due to practical reasons, similar to the BSA. Furthermore, a compact antenna with a well-directed radiation beamwidth is desired for SFCW radar, as this defines the radar's "field of view".

\section{A. Dual Band Rectangular Planar Monopole (RPM)}

The RPM structure is shown in Fig.1. The lower resonant frequency $\left(f_{\mathrm{r}}\right)$ for such monopole can be approximated by equating its area to an equivalent cylindrical monopole of the same length and radius [3]. This $f_{\mathrm{r}}$ is given in eq. (1) as:

$$
f_{r}=\frac{14.4}{L_{s}+h_{1}+h+g_{1}+g_{2}}
$$



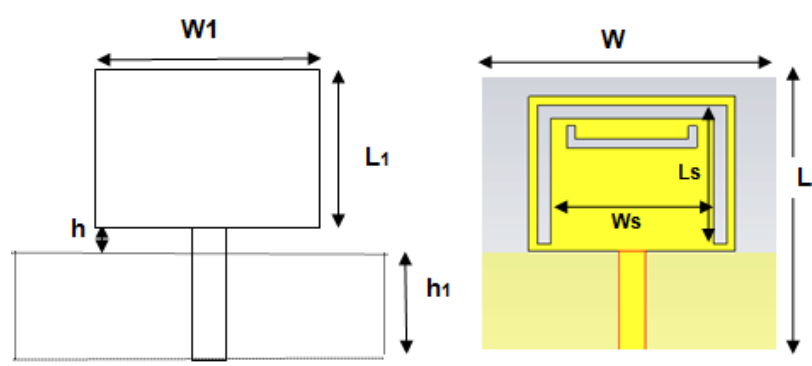

Fig. 1. Structure of rectangular planar monopole and U-slots.

where $g_{1}=A_{1} / 2 \pi h_{1} \sqrt{\varepsilon_{r e}}$ and $g_{2}=A_{2} / 2 \pi L_{1} \sqrt{\varepsilon_{r e}} \cdot A_{1}$ and $A_{2}$ are the areas of the ground plane and radiating plane, respectively, while the effective dielectric constant is given by $\varepsilon_{r e}=\left(\varepsilon_{r}+1\right) / 2$.

The RPM consists of a rectangular radiating patch and feeding line etched on Rogers RT 5880 substrate with a dielectric constant $\left(\varepsilon_{r}\right)$ of 2.2 , thickness $(g)$ of $0.508 \mathrm{~mm}$, loss tangent $(\tan \delta$ ) of 0.009 , and metallization thickness $(t)$ of $0.035 \mathrm{~mm}$. A partial ground plane is printed on the substrate's reverse. Based on eq. (1), the design parameters $L_{l}, h l, h$, and $W_{l}$ are $17.8 \mathrm{~mm}, 11.8 \mathrm{~mm}, 0.2 \mathrm{~mm}$, and 22.8 $\mathrm{mm}$, respectively. The gap $(h)$ between the patch radiator and ground plane introduces coupling capacitance and dictates its wideband behavior.

To effectively communicate the collected radar information to the base station, the antenna design must also support ZigBee operation at $2.45 \mathrm{GHz}$. Etching a Uor inverted-U slot on the radiator introduces this resonance. An approximate equation to determine a single U-slot microstrip patch's operating frequency is proposed in [8], while an inverted-U and $U$ slot's frequency is simplified by approximating the total length of the slot to half the guided wavelength $\left(\lambda_{\mathrm{g}} / 2\right)$ and in eq. (2) [9], with $c=3 \times 10^{8} \mathrm{~m} / \mathrm{s}$ given as the speed of light in vacuum. This structure is shown in Fig.1.

$$
f_{r}=c /\left(2 L_{s}+W_{s}\right)
$$

The addition of a U-slot in the radiating patch also introduces an undesirable third harmonic, which can be compensated by introducing another smaller U-slot, as illustrated in Fig. 1. This antenna's impedance is also influenced by the mutual coupling existence between the two U-slots, thus making their positions on the patch a critical design parameter.

\section{B. CBCPW Fed Bow-Tie Slot Antenna (BSA)}

To design the CBCPW-fed BSA topology, initially a conventional CPW-fed bowtie-slot antenna, as shown in Fig. $2(l)$ is designed. The linearly-polarized bowtie slot antenna is excited by the electric-field lines in the two respective CPW apertures, making the antenna polarization to be along the slot width. The bowtie slot $L_{b}$ determines the central resonance frequency. Lengthening
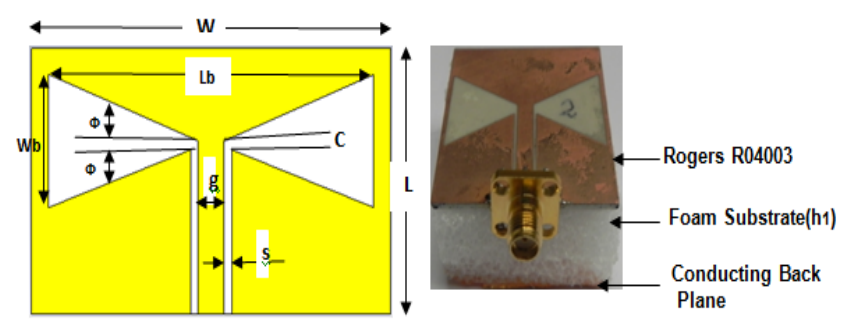

Fig. 2. CPW-fed BSA: (left) conventional, (right) conductor backed (CBCPW-BS1).

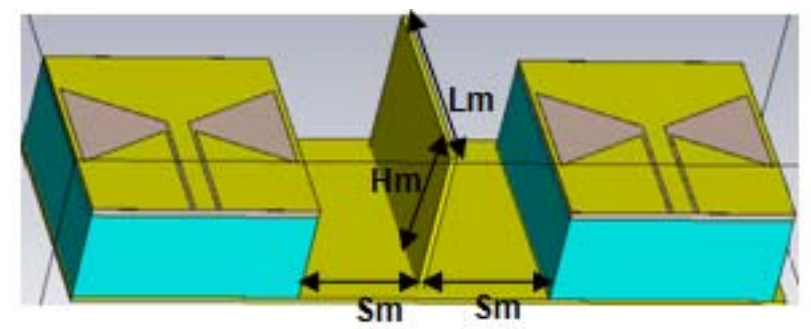

Fig. 3. Simulated pseudo-static configuration for CBCPWBS2.
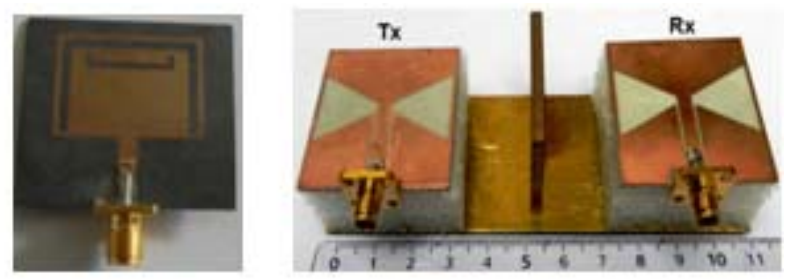

Fig. 4. Fabricated antennas: rectangular monopole (left); and two-antenna configuration (CBCPW-BS2) (right).

$L_{b}$ lowers the resonance. Meanwhile, the bowtie slot width $W_{b}$ tunes the input matching. Increasing $W_{b}$ improves impedance matching, but simultaneously increases the $f_{\mathrm{r}}$. Moreover, flare angle $(\Phi)$ and slot stub with width $(c)$ variation can also improve impedance matching. The antenna is designed on a rectangular substrate dimensioned at $W \times L \mathrm{~mm}$. The substrate is Rogers RO4003 with $\mathcal{E}_{r}=3.48, \tan \delta=0.0027, h=1.524 \mathrm{~mm}$, and $t=0.035 \mathrm{~mm}$. The CPW feed transmission line characteristic impedance is $50 \Omega$, which corresponds to a strip width $g$ of $2.7 \mathrm{~mm}$ and a gap width $s$ of $0.75 \mathrm{~mm}$. Optimized $L_{b}$ and $W_{b}$ are $36.2 \mathrm{~mm}$ and $11.25 \mathrm{~mm}$, respectively.

The conventional bowtie slot antenna exhibits a bidirectional radiation which is contrary to the requirement of the SFCW radar. Conductor-backed, twolayered substrate with distinct permittivities is then introduced on the antenna's rear to reduce back-radiation. A lower dielectric foam substrate with $\mathcal{E}_{r}=1.05$ and $h_{l}=$ $18.5 \mathrm{~mm}$ is used, shown in Fig. 2(r) and referred to as CBCPW-BS1. The main drawback of this antenna is the ideal foam substrate height, $h_{l}$. This $h_{l}$ largely influences the bandwidth and radiation efficiency [10]. A radiator closer to ground decreases radiation efficiency, while 


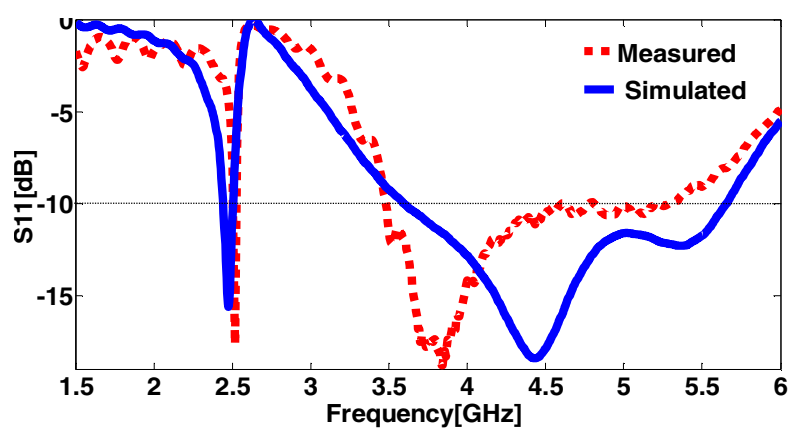

Fig. 5. Measured return loss for the RPM.

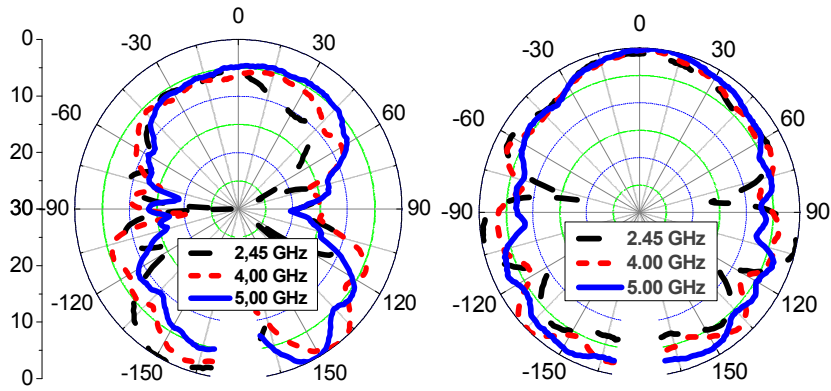

Fig. 6. Radiation patterns for the RPM at: E-plane (left) and H-plane (right).

increasing $h_{l}$ increases the antenna size, and simultaneously reduces the ground plane effect, resulting in a bidirectional radiation pattern once again. In case of this antenna as well, the upper metal layer length $(L)$ is used to optimize the resonance at $2.45 \mathrm{GHz}$, as increasing the antenna length $L$ decreases the resonance frequency. CBCPW-BS1 was fabricated, measured and evaluated in the radar.

\section{Antenna Design for Pseudo Static Radar Configuration}

For the pseudo static radar configuration, it is important to reduce mutual-coupling $\left(S_{21}\right)$ between two nearby antennas to ensure precise target detection. Absorbing material placed between them can facilitate $S_{2 l}$ reductions, besides the conventional method such as inserting a metal plate between transmit and receive antennas. However, the coupling resulting from this plate's usage changes the antennas' return losses $\left(S_{I I}\right)$. Therefore the distance between this metal plate and the antenna $\left(S_{m}\right)$ must be optimal to ensure minimal influence on antennas' $S_{l l}$, while not over-increase the radar size at the same time. Fig. 3 depicts the two-antenna configuration spaced $S_{m}=$ $20 \mathrm{~mm}$ apart from the centered metal plate, referred to as CBCPW-BS2. The optimized metal plate length $\left(L_{m)}\right.$ and height $\left(H_{m}\right)$ based on the requirement of maximal $S_{21}$ reduction from 5.8 to $7 \mathrm{GHz}$ are found to be $39 \mathrm{~mm}$ and $31 \mathrm{~mm}$, respectively.

\section{RESULTS AND DISCUSSION}

The simulation and measurement comparison of each design is presented in this section. Simulations were carried out using CST Microwave Studio using the transient solver. The antenna's return losses were

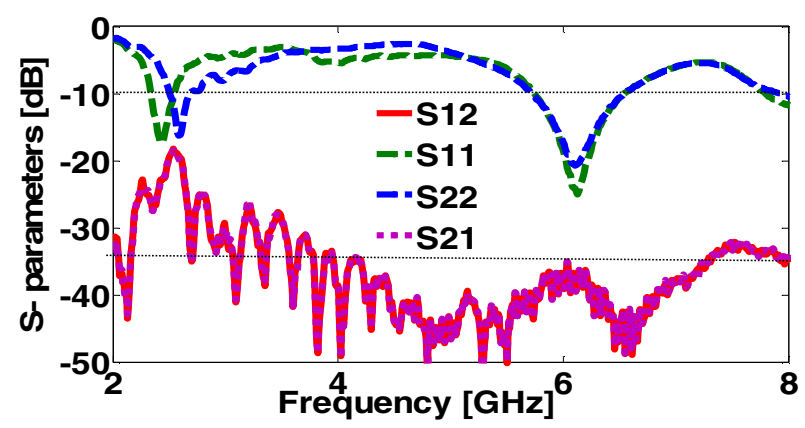

Fig. 7. Measured S-parameters for the CBCPW-BS2.
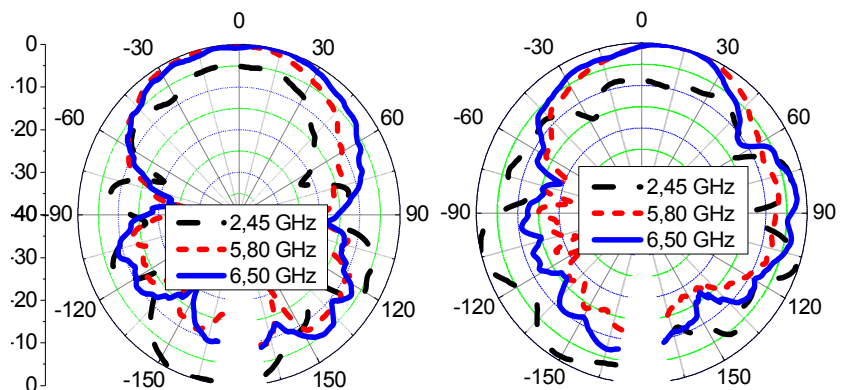

Fig. 8. Radiation patterns for the CBCPW-BS2 at: E-plane (left) and H-plane (right).

measured using a Vector network analyzer (VNA), while radiation pattern measurements were performed in an anechoic chamber.

\section{A. Dual Band Rectangular Planar Monopole Antenna}

The dual-band rectangular planar monopole antenna prototype is shown in Fig. 4(l). Fig. 5 shows the simulated and measured return loss indicating satisfactory performance in meeting the SFCW radar requirements. The measured return loss indicates a resonance shift from $2.45 \mathrm{GHz}$ to $2.50 \mathrm{GHz}$, caused by the slot length fabrication tolerance. The measured co-polarized E-plane and $\mathrm{H}$-plane patterns at $2.45 \mathrm{GHz}, 4.0 \mathrm{GHz}$, and $5.0 \mathrm{GHz}$ are shown in Fig. 6, indicating an omni-directional $\mathrm{H}$ plane. In the E-plane, the measured pattern is similar to a small dipole, producing a bidirectional radiation pattern.

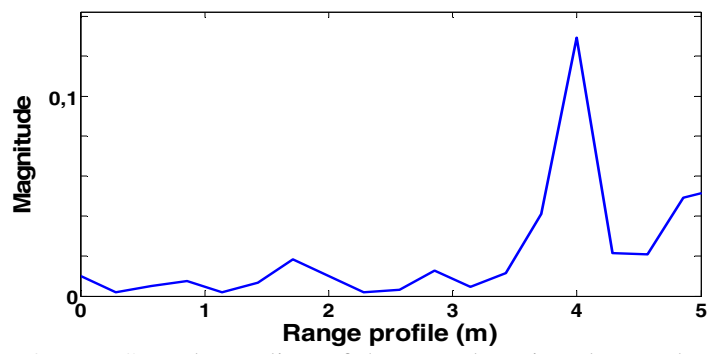

Fig. 9. Sample reading of the PUT location detected using CBCPW-BS2 antenna at $4 \mathrm{~m}$.

\section{B. $C B C P W-B S 2$ Antenna}

As discussed in previous section, CBCPW-BS2 is designed based on CBCPW-BS1, and only the results of CBCPW-BS2 are presented. Fig. 4( $r)$ shows the fabricated CBCPW-BS2 prototype, and its measured $S_{11}$ and $S_{21}$ are depicted in Fig. 7. $10 \mathrm{~dB}$ operating range is observed from 
2.39-2.6 and 5.7-6.85 GHz, while mutual-coupling $\left(S_{21} / S_{12}\right)$ is well below $-37 \mathrm{~dB}$ between 5 and $7 \mathrm{GHz}$. Each radiation pattern is measured by terminating the other antenna using a $50 \Omega$ load. Fig. 8 shows the measured copolarized E- and H-planes. The gain of the both antennas is more than $6 \mathrm{~dB}$ throughout 5.7 to $7 \mathrm{GHz}$ and about $0 \mathrm{~dB}$ from 2.4 to $2.48 \mathrm{GHz}$.

\section{RADAR EVALUATION}

Measurements were performed in a practical environment, i.e., a $5 \times 5 \mathrm{~m}^{2}$ room with antennas fixed on a wall and the person-under-test (PUT) located at different distances from the radar. Performances of the RPM, CBCPW-BS1, and CBCPW-BS2 are evaluated using similar radar. Once the radar is able to locate the position of PUT using one set of antennas, speed and then fall can be detected by the same antennas.

\section{A. Performance Evaluation of RPM}

For RPM prototype, measurements have been taken by separating the two antennas, one transmitting and another receiving, $10 \mathrm{~cm}$ apart. It was not possible to detect the PUT with less than $5 \mathrm{~cm}$ separation due to the high mutual-coupling. As mentioned in previous section, the RPM also features a bidirectional radiation, which is another reason for poor scanning.

\section{B. Performance Evaluation of $C B C P W-B S 2$}

CBCPW-BS2, which consists of two bowties with a common rear conductor plane, and a vertical wall in between, is shown in Fig. 4(r). Using this configuration, the PUT is detectable up to $4 \mathrm{~m}$, as shown in Fig. 9. On the other hand, a perfectly reflecting metal plate has been detected up to $5 \mathrm{~m}$ using this antenna.

TABLE I

RADAR EVALUATION FOR DIFFERENT ANT PROTOTYPES

\begin{tabular}{|l|c|c|c|c|c|c|}
\hline $\begin{array}{l}\text { PUT Distance/ } \\
\text { Prototype }\end{array}$ & $\begin{array}{c}\text { Sm } \\
(\mathbf{c m})\end{array}$ & $\mathbf{1 m}$ & $\mathbf{2 m}$ & $\mathbf{3 m}$ & $\mathbf{4 m}$ & $\mathbf{5 m}$ \\
\hline RPM & 10 & Yes & Yes & No & No & No \\
\hline CBCPW-BS1 & 8 & Yes & Yes & Yes & No & No \\
\hline CBCPW-BS2 & 4 & Yes & Yes & Yes & Yes & Yes \\
\hline
\end{tabular}

\section{Performance Comparison}

All performance evaluations of the designed antennas for different PUT locations form Tx/Rx antennas is show in Table I. It indicates that bow-tie antennas are detecting the PUT till $4 \mathrm{~m}$ distance from the antenna, while the rectangular monopole only yields good results till $2 \mathrm{~m}$ distance. CBCPW-BS2 is performing better than the RPM, mainly due to the high front-to-back ratio and the low mutual-coupling between the bow-tie antennas.

\section{CONCLUSIONS}

Antenna design requirements for SFCW radar have been identified and full-filled by designing two distinct antenna topologies, a rectangular monopole planar antenna and a novel conductor backed CPW-fed bow-tie slot. The optimized dimensions for each type are then fabricated and evaluated using the SFCW radar in pseudostatic configuration. It is concluded that the performance of a conductor backed CPW fed bow-tie slot antenna with a metal plate in between is superior to the rectangular planar monopole antenna topology due to the unidirectional radiation and lower mutual-coupling.

\section{REFERENCES}

[1] M. Mercuri, D. Schreurs, and P. Leroux, "SFCW microwave radar for in-door fall detection," IEEE Topical Conference on Biomedical Wireless Technologies (BioWireless), pp. 53-56, Santa Clara, CA, USA, 15-18 Jan. 2012.

[2] M. Mercuri, D. Schreurs, and P. Leroux, "Investigation of a wireless sensor network for improving in-door fall detection and tag-less localization," European Conference on the Use of Modern Information and Communication Technologies (ECUMICT), pp. 261-269, 2012.

[3] K. G. Thomas, and M. Sreenivasan, "A simple ultrawidebandplanar rectangular printed antenna withband dispensation," IEEE Trans. on Antennas and Propagation, vol. 58, No. 1, Jan.2010.

[4] A. Kumar and N. Gunasekaran, "A new novel compact planar UWB antenna," Proceedings of International Conference on Signal Processing, Communication, Computing and Networking Technologies, pp. 41-45, July 2011.

[5] M. E. Bialkowski and A. M. Abbosh, "Design of UWB planar antenna with improved cut-off at the out-of-band frequencies," IEEE Antennas and Wireless Propagation Letters, vol. 7, pp. 408-410, 2008.

[6] E. S. Angelopoulos, A. Anastopoulos, C. E. Githonas, and D. I. Kaklamani, "A modified bow-tie slot antenna fed by a CPW-to-CPW transition loaded with inductively coupled slots for Ultra-Wideband applications," IEEE International Workshop on Antenna Technology (IWAT), pp. 513-516. March 2005.

[7] F. Lu, Q. Feng, and S. Li, "A novel CPW-fed bow-tie slot antenna for $5.8 \mathrm{GHz}$ RFID tags," Progress In Electromagnetics Research Symposium (PIERS), pp. 327300, July 2008.

[8] R. Bhalla and L. Shafai, "Resonance behavior of single uslot and dual u-slot antenna," IEEE International Symposium Antennas and Propagation Society, vol. 2, pp. 700-703, July 2001

[9] W.-S. Lee, D.-Z. Kim, K.-J. Kim, and J.-W. Yu, "Wideband planar monopole antennas with dual band-notched characteristics," IEEE Trans. on Microwave Theory and Techniques, vol. 54, no. 6, pp. 2800-2806, June 2006.

[10] J. P. Jacobs, J. Joubert, and J. W. Odendaal, "Radiation efficiency and impedance bandwidth of conductor-backed CPW-fed broadside twin slot antennas on two-layer dielectric substrate," IEE Proceedings on Microwave, Antennas and Propagation, pp. 185-190, Aug. 2003. 